\title{
Discriminated conditioned suppression in rats
}

\author{
Thais Arantes Ribeiro, Edson Massayuki Huziwara, Tathianna Amorim Souza Montagnoli and \\ Deisy das Graças de Souza \\ Universidade Federal de São Carlos, São Carlos, SP, Brazil
}

\begin{abstract}
This experiment evaluated the effects of superimposing the Estes-Skinner Conditioned Emotional Response (CER) procedure on one of two components of a multiple schedule. The question was whether CER conditioning occurred under contextual control. The procedure had four experimental phases: (1) baseline of operant responding under a two-component multiple schedule (mult VI 30 VI 30), one component correlated with the house light on and the other correlated with the house light off (light/ dark components), (2) introduction of tone-shock pairings during the light component only, (3) return to baseline contingencies, and (4) reintroduction of the tone (but not shock) in the light component. Three Wistar rats showed robust suppression of responding in the light component, and the suppression also partially generalized to the dark component. The suppression was stronger during the pre-aversive stimulus than during the intervals immediately before and after its presentation. Responding partially recovered under baseline contingencies, but response rates remained lower in the light component than in the dark component. Thus, under the present experimental conditions, the context in which CER conditioning occurred (i.e., the house light-illuminated chamber) also produced conditioned suppression, and contextual control of suppressed responding generalized to another context, one that shared some but not all elements of the first context (i.e., the same chamber not illuminated by a house light). These results have direct implications for our understanding of emotional conditioning produced in the laboratory and for analysis of related phenomena addressed in the clinic. Keywords: conditioned suppression; aversive control; contextual control; tone-shock pairings; rats.
\end{abstract}

Received 2 October 2011; received in revised form 21 February; accepted 21 February 2012. Available online 29 June 2012.

\section{Introduction}

In the Estes-Skinner "anxiety" or Conditioned Emotional Response (CER) paradigm, a neutral preaversive stimulus is paired with an aversive stimulus (e.g., electric shock to the feet) during a baseline of operant behavior (e.g., bar press). The typical effect, depending on several parameters (c.f., Blackman, 1977), is the suppression of the ongoing operant behavior during the pre-aversive (or "warning") stimulus and its recovery after the presentation of the aversive stimulus (the "safe" period).

The original procedure used by Estes and Skinner (1941) trained rats to press a lever for food under a fixed interval (FI) schedule of reinforcement. When the baseline of FI responding reached a stable state (Sidman, 1960), a pre-aversive stimulus (e.g., tone) was presented for $3 \mathrm{~min}$, at the end of which a brief

Thais Arantes Ribeiro, Edson Massayuki Huziwara, Tathianna Amorim Souza Montagnoli and Deisy das Graças de Souza, Departamento de Psicologia, Universidade Federal de São Carlos. Correspondence regarding this article should be directed to: Thaís Arantes Ribeiro or Deisy de Souza, Departamento de Psicologia, Universidade Federal de São Carlos, Via Washington Luís, Km 235, 13.565-905, São Carlos, SP, Brazil. E-mail: thais.ribeiro13@gmail.com or ddgs@ufscar.br electric shock was delivered through the grid floor of the experimental chamber. Experimental sessions were $6 \mathrm{~h}$ long with two tone-shock pairings during each hour. Later, the pre-aversive stimulus was increased to $5 \mathrm{~min}$, and the tone-shock pairings occurred only once during each hour. All subjects showed a complete suppression of responding during the pre-aversive stimulus by the third or fourth session. Bar presses recovered immediately after the presentation of the electric shock. The conditioned suppression effect (or CER) has been replicated in several subsequent experiments using different species, responses, and aversive stimuli (e.g., Blackman, 1968; Blackman \& Scruton, 1973; Burdick \& James, 1970; Carlton \& Didamo, 1960; Houten, O'Leary, \& Weiss, 1970; James \& Mostoway, 1968; Nascimento \& Carvalho Neto, 2011; Reberg \& Memmott, 1979; Stein, Sidman, \& Brady, 1958; Weiss, 1968 ), but its magnitude depends on several parameters. For example, Stein et al. (1958) found that the most important determinant of suppression was the ratio of the duration of the conditioned stimulus (CS) to the between-stimulus interval.

The conditioned suppression paradigm involves discrimination between "warning periods" in the presence of the pre-aversive stimulus and "safe" periods in the absence of the CS. Recovery of responding often 
observed after the presentation of the aversive stimulus suggests that the suppressive effects could be confined to the warning periods. However, several studies found a reduction in overall response rates, suggesting that, to some degree, the entire context in which the aversive stimulus is presented could acquire some aversive function. Thus, one important issue is whether the context also produces conditioned suppression when CER conditioning occurs in a "context".

Relationships between conditioned suppression and stimulus control have been investigated in some experiments, but the research addressed other relationships among conditioned suppression and discrimination learning. For example, an experiment conducted by Hearst (1965) used a multiple schedule of reinforcement initially composed of two alternating components. In the $\mathrm{S}^{\mathrm{D}}$ component not signaled by any programmed external stimulus, lever-pressing responses were reinforced by food pellets according to a VI 1-min schedule. In the $\mathrm{S}^{\Delta}$ component signaled by a flashing light for half of the rats and a tone for the other half, lever-pressing was not reinforced (extinction). Either the $\mathrm{S}^{\mathrm{D}}$ or $\mathrm{S}^{\Delta}$ components were in effect during successive 1-min periods according to a nonsystematic order.

After response rates became stable, a third component was added to the multiple schedule. In this new component, named the CER, responses were reinforced by food pellets in a VI 1-min schedule. The CER component was signaled by a flashing light for subjects exposed to the tone in the $\mathrm{S}^{\Delta}$ components and a tone for the subjects where a flashing light was the stimulus in the $\mathrm{S}^{\Delta}$ components. The session was composed of $1 / 6 \mathrm{CER}$ components, $1 / 6 \mathrm{~S}^{\Delta}$ components, and $4 / 6 \mathrm{~S}^{\mathrm{D}}$ components presented in a semi-random order with the only restriction that each CER component should be preceded and followed by $\mathrm{S}^{\mathrm{D}}$ components. This condition was maintained for six sessions, and the rate of responding was measured separately in each component of the multiple schedule. Subjects continued to respond at high rates during the $\mathrm{S}^{\mathrm{D}}$ component and at low rates during the $\mathrm{S}^{\Delta}$ component. In the CER component, all subjects emitted lever-press responses, but variability was observed in response rates. In the next phase, an unavoidable shock was presented at the end of each CER period, and the tone or flashing light, initially an unconditioned stimulus, acquired the function of a pre-aversive stimulus. Introduction of the shock produced response suppression during the CER components, and response rates also decreased during the $S^{D}$ components and increased during the $S^{\Delta}$ components. The subjects began to respond in the $\mathrm{S}^{\Delta}$ component, although responses in this component had never been previously reinforced. According to Hearst, this response pattern could be considered a disruption of the stimulus control established in the previous phases.

Weiss (1968) conducted an experiment that sought to systematically replicate the procedure used by Hearst (1965). The results, however, were different from those obtained in the original experiment. Whereas
Hearst found an increase in responding during the $\mathrm{S}^{\Delta}$ components, Weiss found a decrease in the rats' responses in both the $\mathrm{S}^{\mathrm{D}}$ and $\mathrm{S}^{\Delta}$ components. The results described in further studies were similar to those described by Weiss, replicating the general decrease in response rates in all of the components during the sessions (Blackman \& Scruton, 1973) instead of the disruption in stimulus control previously observed.

In these previous studies, the goal was to produce different patterns of responding (i.e., high rates in the VI component and low rates in the extinction component) and then observe the eventual effects of the tone-shock pairings (e.g., Blackman \& Scruton, 1973; Hearst, 1965; Weiss, 1968) in an independent component of the multiple schedule. This manipulation was justified, considering that the main interest of these experiments was to describe the possibly deleterious effects of the presentation of an aversive stimulus on discriminated responding.

The present study took a different approach, determining whether similar patterns of responding maintained by two components of a multiple schedule (programmed with the same reinforcement contingencies) are differentially affected by the superimposition of the CER procedure on only one of them. We examined whether the eventual CER effects are restricted to the component in which the CER is introduced (i.e., contextual control) or whether these effects generalize to the component in which no direct pairing of a neutral and aversive stimulus occurs.

We used a two-component multiple schedule with each component correlated with a different exteroceptive stimulus (Catania, 1998; Reynolds, 1961) but with equal VI schedules that operated in each of them. The reinforcement contingencies should result in an equal distribution of responses across both components (Catania, 1998; Herrnstein, 1970). One component of the multiple schedule was correlated with the presence of a white light, and the other component was correlated with the absence of the light (for convenience, hereafter the components are named "light" and "dark", respectively). The pre-aversive stimulus was a tone, and the aversive stimulus was an electric shock delivered through the grid floor.

\section{Methods}

\section{Subjects}

Three naive male Wistar rats (Rattus norvegicus) approximately 90 days old at the beginning of the experiment were used. Rats were housed in individual cages and water-deprived for $23 \mathrm{~h}$ before the experimental sessions. Food was continuously available. The temperature was controlled between $18^{\circ} \mathrm{C}$ and $25^{\circ} \mathrm{C}$ under a $12 \mathrm{~h} / 12 \mathrm{~h}$ light/dark cycle in the colony room. All of the conditions were in accordance with the ethical principles for the use of laboratory animals established by the Brazilian Science Society in Laboratory Animals (SBCAL). The study was approved by the Ethics Committee in Animal 
Experimentation (CEEA) of the Federal University of São Carlos (protocol no. 023/09).

\section{Apparatus}

A standard rat chamber (ELT-02 Eltrones) served as the experimental enclosure. The chamber was equipped with a response lever located on the right wall, and a dipper was located at the floor level below the lever. The grid floor had 13 metal bars through which electric shocks were delivered. A $24-\mathrm{V}$ direct-current yellow light was located above the bar. Two speakers were located on the front wall near the floor at both sides of the response bar. The experimenter controlled the session duration and presentation of the stimuli. Responses and reinforcers were recorded in 10-s intervals.

\section{Procedure}

The experimental sessions were conducted 7 days per week and lasted $40 \mathrm{~min}$ each. Before the experiment, the rats were weighed daily and had access to water for $1 \mathrm{~h}$ until their body weights stabilized. After achieving a stable weight, the subjects received dipper training, and lever press responding was shaped through successive approximations and maintained under a continuous reinforcement schedule until 100 responses were reinforced.

The experimental design included four phases: (1) baseline of operant responding under a VI VI multiple schedule with equal schedules (mult VI $30 \mathrm{~s}$ VI $30 \mathrm{~s}$ ) correlated with the presence or absence of the light (lightdark components), (2) introduction of two tone-shock pairings per session during the light components only, (3) return to baseline conditions, and (4) presentation of the tone alone (without the electric shock).

Phase 1: Baseline-Multiple Variable Interval Schedules (mult VI VI). In this phase, bar presses were initially reinforced according to a single VI schedule. The mean interval gradually increased from 5 to $30 \mathrm{~s}$. The 30-s VI was programmed with 11 intervals (Fleshler \& Hoffman, 1962) sorted in three different sequences. The first response after each interval had elapsed activated the dipper and provided access to $0.2 \mathrm{ml}$ of water.

After stable responding under the VI $30 \mathrm{~s}$, the procedure changed to a multiple schedule with two components (mult VI 30 VI 30). One component was correlated with the onset of the yellow light (i.e., light component). The other component was correlated with the light off (i.e., dark component). The component duration was $2 \mathrm{~min}$. Each session had 10 light and 10 dark components that alternated in a semi-random order, and one component could not be in effect more than three consecutive times.

The baseline phase was in effect until the stability criterion was reached. Stability was defined as a variation of less than $20 \%$ of the average number of responses across five consecutive sessions for each component.

Phase 2: Tone-shock pairings superimposed on the light component of the multiple schedule. This phase was in effect for 10 sessions. Figure 1 presents a schematic representation of the procedure with the sequence of events throughout the session. Two CS-unconditioned stimulus (US) pairings were presented in each session. The tone duration was $5 \mathrm{~s}$, and the tone was turned off simultaneously with the beginning of the electric shock. The shock intensity was $0.4 \mathrm{~mA}$ and the shock duration was $0.5 \mathrm{~s}$. The CS-US pairings were presented in $2 / 10$ presentations of the light component, one in the first half of the session (during the $18^{\text {th }}$ minute) and the other in the second half(during the $34^{\text {th }}$ minute). Given the distribution of the multiple schedule components, the tone-shock pairing could occur at different temporal distances from the beginning of the component (see black bars in Figure 1). Only the baseline contingencies were in effect during the remaining presentations of the light component and in all of the presentations of the dark component.

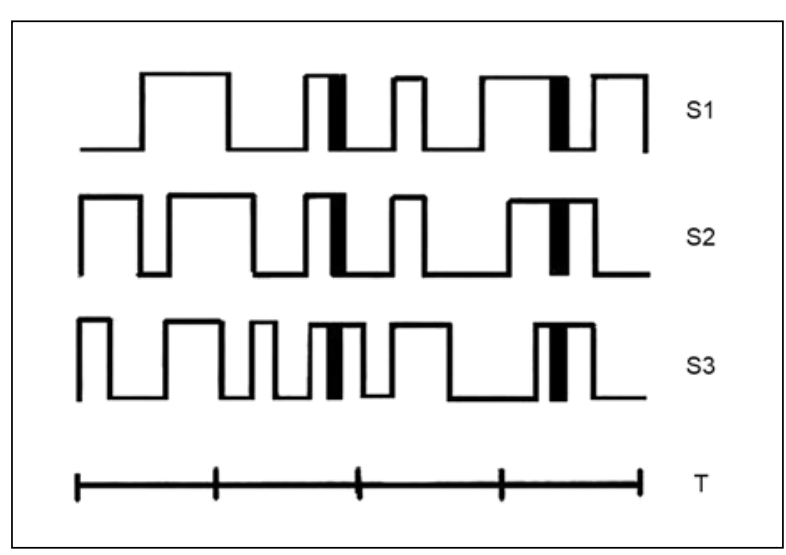

Figure 1. Schematic representation of the sequence of events in a session with tone-shock pairings (Phase 2). $T$ indicates the passage of time in minutes. $S 1, S 2$, and $S 3$ correspond to different sequences of presentation of the multiple-schedule components (light on/light off) across the session. The horizontal lines indicate which component was in effect: (0) dark or (1) light. Black bars represent the toneshock pairings (during the $18^{\text {th }}$ and $34^{\text {th }}$ minutes of each session). The component duration was $120 \mathrm{~s}$, and the same component could be presented up to three times in sequence.

Phase 3: Return to baseline (mult VI 30 s VI 30 s). Phase 3 was identical to Phase 1 and in effect for three sessions. The purpose of this phase was to observe the course of response rates after the interruption of the CER procedure.

Phase 4: Presentation of pre-aversive stimulus without shock. The fourth phase, conducted in two additional sessions, evaluated whether the preaversive stimulus (i.e., tone) had acquired any aversive function after being paired with the electric shock and whether these effects persisted after the three baseline sessions. The procedure was the same as Phase 2 with the exception that the electric shock presentation was omitted at the end of the tone presentation during the $18^{\text {th }}$ and $34^{\text {th }}$ minutes.

\section{Results}

Figure 2 presents the overall response rates in each component of the VI VI multiple schedule across the 
experimental sessions and phases. Black lines represent responding in the dark component, and gray lines represent responding in the light component.

In the initial baseline, beginning with the introduction of the multiple schedules, all three rats reached the stability criterion within six to nine sessions. For each subject, response rates $(\mathrm{R} / \mathrm{min})$ were similar across the two components of the multiple schedule. The rates were slightly higher for R2 than for the other two subjects. The averages in the last three sessions in the light and dark components were 10.0 and 8.6 for R1, 16.4 and 16.5 for R2, and 9.7 and 10.7 for R3, respectively. In Phase 2, when the tone- shock (CS-US) pairings were superimposed on the light component, response rates decreased abruptly in both components for all three rats. However, response suppression was stronger in the light component than in the dark component throughout most of the sessions. The only exceptions occurred in the first two sessions for R1 and R3, in which the rates in both components showed the same amount of decrease. R1 and R2 showed a slight recovery in response rates across the 10 sessions of this phase, but the rates remained lower than during the initial baseline. For R3, responding in the light component was almost completely suppressed across the 10 sessions.

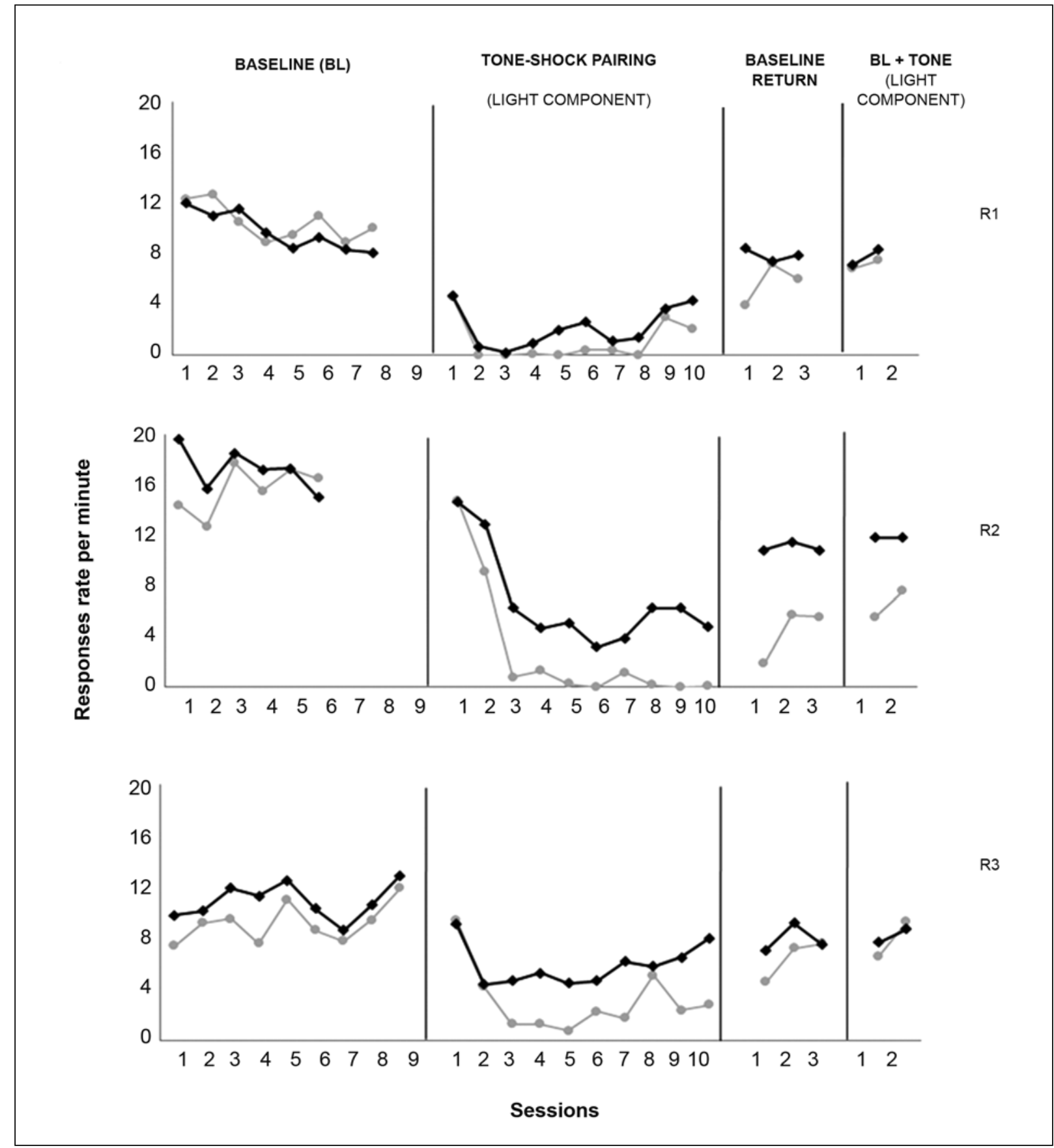

Figure 2. Response rates $(\mathrm{R} / \mathrm{min})$ for individual subjects across the four phases of the experiment. Gray lines indicate responding in the light component, and black lines indicate responding in the dark component. 
After the interruption of the suppression procedure, in the three sessions of Phase 3 (i.e., return to baseline), response rates increased immediately in both components for subjects R1 and R2 but remained lower in the light component than in the dark component. For $\mathrm{R} 3$, the rates increased in the light component, whereas responding in the dark component remained stable at the same rate as in the previous sessions. The rates became the same in the two components by the third session. The mean response rates in these three sessions in the light and dark components were 5.7 and 7.9, 4.4 and 11.0 , and 6.5 and 7.9 for the three subjects, respectively.

Phase 4 evaluated the effects of introducing the tone alone in the light component. The rates of responding remained almost the same as in the previous baseline across the two sessions of this phase.

In Figure 3, local response rates $(\mathrm{R} / \mathrm{s})$ in the 10 -s intervals immediately before and after each CS-US pairing were contrasted by the overall response rates in the dark component and during the entire baseline sessions in Phases 1 and 3 in both components. White bars represent baseline rates. In the upper panel for the light component, gray bars represent response rates in Phase 2 in the intervals before and after the CSUS pairings, and black bars represent the rates in the 10-s interval during which the CS-US pairings were presented. For the dark component (bottom panel), the gray bars represent overall response rates in Phase 2 .

The average data in this figure confirm the trends observed across individual sessions shown in Figure 2. Response rates were much higher during the initial baseline than during any other experimental phase. Introduction of the CER procedure affected responding in both components of the multiple schedule, but the effects were stronger in the light component that was correlated with the CS-US pairings. In this component, the suppression was stronger during the tone-shock intervals (black bars) than in the pre- and post-shock intervals for all three subjects. Responding recovered during the final baseline but remained lower than during the initial baseline, and recovery was greater in the dark component.

\section{Discussion}

Rats were initially exposed to a multiple schedule of reinforcement with equal VI schedules, each signaled by a different stimulus condition (Catania, 1998; i.e., light and dark [light off]). The schedule contingencies established stable and similar response patterns in both components for the three rats. A CER conditioning procedure was then superimposed on only one of the components of the multiple schedule (i.e., the light component). The multiple VI VI procedure allowed us to compare responding when the CER was in effect (i.e., light on) or was not in effect (i.e., dark) during the same session. The ABAC reversal design (baseline, baseline + CER, baseline, baseline + CS) allowed comparisons across the experimental phases. Finally, recording the bar presses in 10-s intervals allowed us to analyze local behavioral patterns in the intervals that preceded and followed the CS-US pairings and during the interval in which the pairings occurred (Figure 3).

After the indiscriminate responding during the initial baseline, all three subjects showed differential responding during the two components when the signaling aversive stimulus was introduced. A suppression of responding occurred in both components, but the suppressive effects were more pronounced during the presentations of the light component (Figure 2). As soon as the light that signaled the component was presented, the subjects decreased their response rates. Additionally, when the pre-aversive stimulus (i.e., tone) was turned on in the presence of light, all subjects responded at a very low rate or did not respond at all (see black bars in top panel of Figure 3). Responding partially recovered when the tone was turned off simultaneously with the shock presentation in the light component (see gray bars at right of dark bars in Figure 3). Although at lower rates than during baseline, responding increased when the light component alternated to the dark component (see gray bars in bottom panel of Figure 3).

Several aspects of these results deserve consideration. First, responding in the light component replicated the typical effects of CER conditioning (Blackman, 1968; Blackman \& Scruton, 1973; Carlton \& Didamo, 1960; Houten et al., 1970; Nascimento \& Carvalho Neto, 2011; Reberg \& Memmott, 1979; Stein et al., 1958; Weiss, 1968). The replication adds to the generality of the CER paradigm and provides evidence that, although incorporated into a complex schedule, the procedure reliably produced the typical suppression of responding during the CS and response recovery to some degree after the presentation of the aversive stimulus. However, these findings do not support the suggestion that lever pressing would be suppressed in the presence of the pre-aversive stimulus only if such a behavioral pattern did not reduce the opportunities to obtain reinforcement (Stein et al., 1958). In the present experiment, subjects showed a total (R1 and R2) or partial (R3) suppression of lever presses in the light component in Phase 2, losing at least half of the programmed reinforcers in that component, although the opportunities to obtain reinforcement did not change (i.e., food continued to be available according to the VI schedule). The role of other variables, however, such as shock intensity, shock duration, and their combination, should also be investigated.

The production of reliable CER effects in the light component was important for the analysis of what occurred to responding in the other component of the multiple schedule. Data suggest that the subjects discriminated between the dark and light components and that discrimination was based on the presence $v s$. absence of the aversive stimulus because the reinforcement contingencies were otherwise the same under both components. The discrimination, however, was partial because the tone-shock pairings, presented 


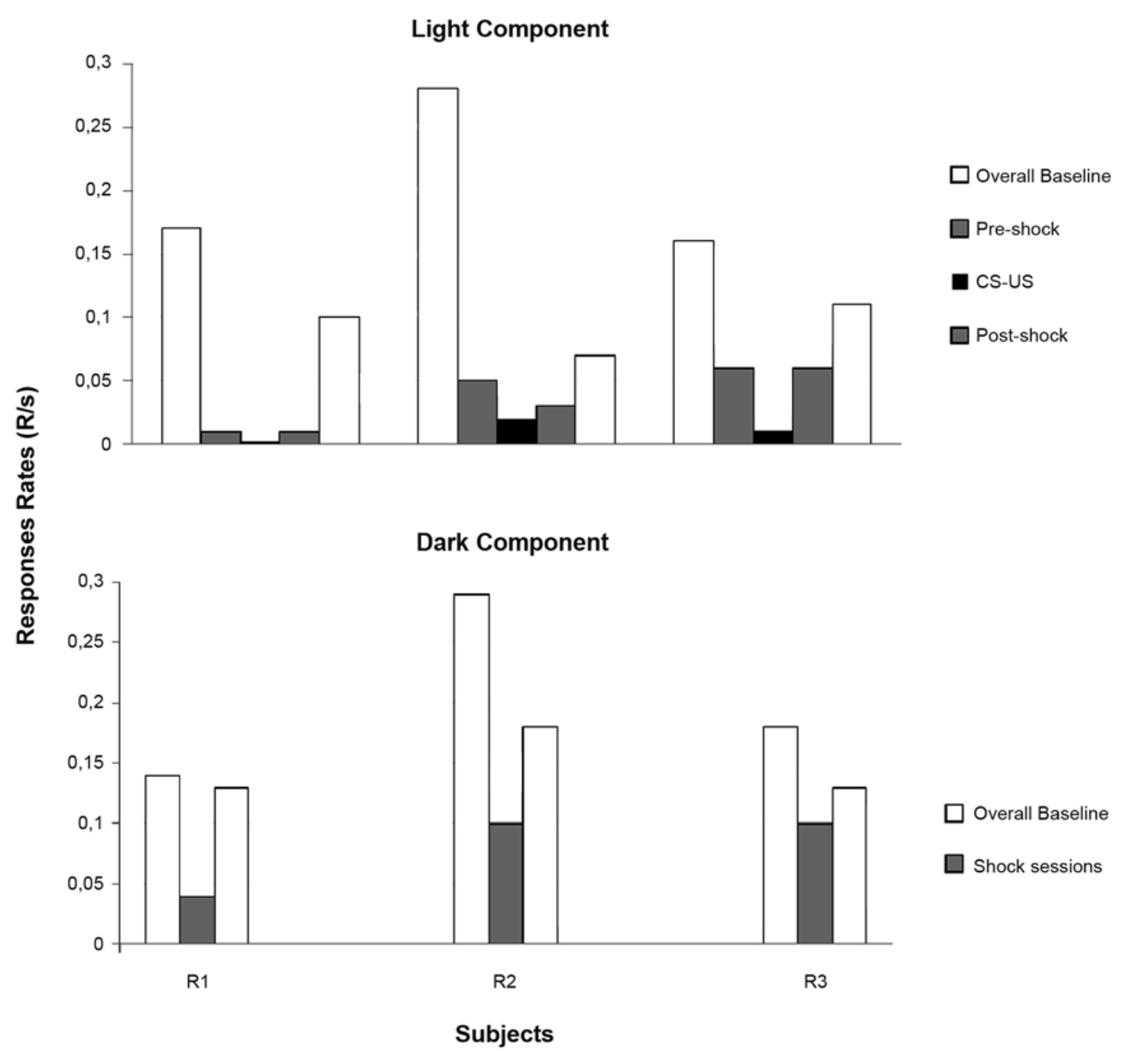

Figure 3. Mean response rates $(\mathrm{R} / \mathrm{s})$ for individual rats. White bars represent overall rates during the baselines (Phases 1 and 3 ). Gray bars represent response rates in the 10-s interval before and after the CER (Phase 2). Black bars represent response rates during the presentation of the tone.

only in the light component, also affected behavior in the dark component. Thus, subjects exhibited behavioral patterns generally described as "anxiety" in both components of the multiple schedule, although with greater intensity in the light component. This indicates that it was not restricted to this situation, despite the contextual control over response suppression by a specific situation (i.e., the presence of the house light plus occasional occurrences of tone + electric shock presentations); instead, it showed some generalization to the dark component.

These results support the broad notion that an aversive event that occurs in a specific context may also produce emotional behavior, escape and avoidance responses in other contexts, although to a lesser extent than in the original situation.

According to Bouton and King (1983), the stimuli that comprise the background in which learning occurs are importantly involved in conditioning and learning.
Despite little agreement about how to conceptualize the role of context in learning, an important unanswered question is whether contextual control functions as independent second-order conditional stimuli or simply as elements of compound first-order conditional and discriminative stimuli (Bush, Sidman, \& de Rose, 1989).

Estes (1973; see also Bouton \& King, 1983) suggested that contexts retrieve or carry information about relationships among events that occur within them, without establishing direct associations with them. Some research focused on another aspect of contextual control, suggesting that it plays an important role in the recovery of behavior after extinction (Bouton, 1986, 1993, 2002; Neumann, 2006).

Our findings on conditioned suppression suggest that the contextual control exerted by the light was extended to another context (i.e., the dark component) that shared some but not all elements of the first context. 
For example, both schedules were in effect in the same experimental chamber with all of its specific features where the subjects were placed during the 40-min session. For the rest of the day, subjects remained in their home cages. Bar presses were reinforced in both "environments", and these environments alternated within short periods of time. As reported by Burdick and James (1970), some spontaneous recovery from the extinction of the conditioned suppression of licking by rats appeared to have been a function of conditioning to the apparatus cues. Under the present conditions, however, we could not unequivocally determine whether the light exerted contextual control as a "higher-order" conditional stimulus or as a compound (associated with pre-aversive stimulus) first-order conditional and discriminative stimulus.

The results also show that contextual cues can establish discrimination between dark and light components in rats, differentially affecting response rates in the components in which the aversive stimulus was or was not directly paired. The fact that the suppression effect was stronger in the light component than in the dark component suggests that identifying which stimuli control "anxious" behavior and with which environments these stimuli may have been paired could be possible in basic or applied settings.

Another aspect to consider is the long-lasting disruptive effect of tone-shock presentations on responding in both components of the multiple schedule. None of the rats showed a total recovery of response rates when the procedure returned to baseline conditions in Phase 3. Subject R1, for example, pressed the lever an average of $9.3 \mathrm{R} / \mathrm{min}$ (10 and 8.6) in the last sessions of Phase 1 but no more than $6.9 \mathrm{R} / \mathrm{min}$ (5.7 and 7.9) in Phase 3. Remaining to be determined is whether an extension of Phase 3 would result in further increases in response rates with a return to the initial baseline rates. This was not done in the present study because we wanted to verify the effects of the presentation of the tone alone in Phase 4.

The present results have direct implications for our understanding of emotional conditioning produced in the laboratory and for the exploration of potentially relevant variables in applied settings.

Ultimately, new studies must be conducted in basic and applied research to further clarify these issues. In the basic research field, evaluating, for example, (1) whether the presentation of a shock without a signaling stimulus can generate differential contextual control over behavior; (2) whether the modality of the aversive stimulus other than the electric shock produces different effects on the degree of suppression and contextual control; (3) whether manipulations of the ratio between safe and unsafe periods affect the results in the same direction as predicted by Carlton and Didamo (1960), (4) whether environmental similarity, defined in terms of the amount of common aspects between the components in which the aversive stimulus is or is not presented plays any role in establishing contextual control, and
(5) the effects of the number and order of contexts on discrimination performance.

With regard to applied research, investigating whether the conclusions drawn from this study can be generalized to contexts in which the presence of aversive stimulation is inevitable or recurrent could be relevant (e.g., in hospitals or dental clinics).

\section{Acknowledgments}

The National Council of Science and Technology (CNPq) supported this research (scholarship to T.A.R.). The authors are members of the National Institute of Science and Technology on Behavior, Cognition, and Teaching, supported by FAPESP (grant no. 08/57705-8) and CNPq (grant no. 573972/2008-7). Portions of this paper were presented at the 18th Annual Meeting of the Brazilian Association of Psychotherapy and Behavioral Medicine (ABPMC), Campinas, 2009. The authors thank two anonymous reviewers for their valuable comments and suggestions that greatly improved the paper.

\section{References}

Blackman, D. (1968). Response rate, reinforcement frequency, and conditioned suppression. Journal of the Experimental Analysis of Behavior, 11, 503-516.

Blackman, D. (1977). Conditioned suppression and the effects of classical conditioning on operant behavior. In W. K. Honig \& J. E. R. Staddon (Eds.), Handbook of operant behavior (pp. 340-363). Englewood Cliffs, NJ: Prentice-Hall.

Blackman, D. E., \& Scruton, P. (1973). Conditioned suppression and discriminative control of behavior. Animal Learning and Behavior, $1,90-92$.

Bouton, M. E. (1986). Slow reacquisition following the extinction of conditioned suppression. Learning and Motivation, 17, 1-15.

Bouton, M. E. (1993). Context, time, and memory retrieval in the interference paradigms of Pavlovian learning. Psychological Bulletin, 114, 80-99.

Bouton, M. E. (2002). Context, ambiguity, and unlearning: Sources of relapse after behavioral extinction. Biological Psychiatry, 52, 976-986.

Bouton, M. E., \& King, D. A. (1983). Contextual control of the extinction of conditioned fear: Tests for the associative value of the context. Journal of Experimental Psychology: Animal Behaviour Processes, 9, 248-265.

Burdick, C. K., \& James, J. P. (1970). Spontaneous recovery of conditioned suppression of licking by rats. Journal of Comparative and Physiological Psychology, 72, 467-470.

Bush, K. M., Sidman, M., \& de Rose, T. (1989). Contextual control of emergent equivalence relations. Journal of the Experimental Analysis of Behavior, 51, 29-45.

Carlton, P. L., \& Didamo, P. (1960). Some notes on the control of conditioned suppression. Journal of the Experimental Analysis of Behavior, 3, 255-258.

Catania, A. C. (1998). Learning, 4th edition. Upper Saddle River, NJ: Prentice-Hall.

Estes, W. K. (1973). Memory and conditioning. In F. J. McGuigan \& D. B. Lumsden (Eds.), Contemporary approaches to conditioning and learning (pp. 265-286). Washington, DC: V.H. Winston.

Estes, W. K., \& Skinner, B. F. (1941). Some quantitative properties of anxiety. Journal of Experimental Psychology, 29, 390-400.

Fleshler, M., \& Hoffman, H. S. (1962). A progression for generating variable-interval schedules. Journal of the Experimental Analysis of Behavior, 5, 529-530.

Hearst, E. (1965). Stress-induced breakdown of an appetitive discrimination. Journal of the Experimental Analysis of Behavior, 8, 135-146.

Herrnstein, R. J. (1970). On the law of effect. Journal of the Experimental Analysis of Behavior, 13, 243-266. 
Houten, R. V., O’Leary, K. D., \& Weiss, S. J. (1970). Summation of conditioned suppression. Journal of the Experimental Analysis of Behavior, 13, 75-81.

James, J. P., \& Mostoway, W. W. (1968). Conditioned suppression of licking as a function of shock intensity. Psychonomic Science, 13, $161-162$.

Nascimento, G. S., \& Carvalho Neto, M. B. (2011). Supressão condicionada com diferentes estímulos aversivos: choque elétrico e jato de ar quente [Conditioned suppression with different aversive stimuli: electric shock and hot air blast]. Acta Comportamentalia, 19, 269-280.

Neumann, D. L. (2006). The effects of physical context changes and multiple extinction contexts on two forms of renewal in a conditioned suppression task with humans. Learning and Motivation, 37, 149-175.
Reberg, D., \& Memmott, J. (1979). Shock as a signal for shock or no shock: a feature-negative effect in conditioned suppression. Journal of the Experimental Analysis of Behavior, 32, 387-397.

Reynolds, G. S. (1961). Attention in the pigeon. Journal of the Experimental Analysis of Behavior, 4, 203-208.

Sidman, M. (1960). Tactics of scientific research: Evaluating experimental data in psychology. New York: Basic Books.

Stein, L., Sidman, M., \& Brady, J. V. (1958). Some effects of two temporal variables on conditioned suppression. Journal of the Experimental Analysis of Behavior, 1, 153-162.

Weiss, K. M. (1968). Some effects of the conditioned suppression paradigm on operant discrimination performance. Journal of the Experimental Analysis of Behavior, 11, 767-775. 